\title{
Disadvantages of dental implant
}

\author{
Do Hun SONG, So Min KIM, Kyung Sook HWANG*
}

Dept. of Dent. Tech., Shin Han Univ., Korea

The main discourse: 1 . Implants are very weak in taste and sensation. The current implant fixture is in the form of making a screw hole in the alveolar bone and inserting it there. Due to the nature of the artificial tooth, it cannot reproduce the periodontal ligaments and nerve nerves found in natural teeth. Because of this, they do not feel the texture and taste, and they do not feel their excessive masticatory force, which leads to fracture of the implant, and pain in the temporomandibular joint is often felt after eating.

2. There is no periodontal ligament around the implant fixture, and because nerves and blood vessels are cut off, sudden extraction often occurs after a certain period of time because it cannot withstand long mechanical masticatory force.

3. Also, if you do not take care of it regularly, plaque and tartar are formed along the screw line, and the inflammatory factor can penetrate deep into the bone.

4. Currently, under the National Health Insurance Act, only two implant insurances are applied to the elderly over 65 years of age. The benefits of health insurance are still insufficient, and since all teeth have 4 teeth with the same function, top, bottom, left, and right, at least 4 insurances must be made. Moreover, additional application is necessary for the disabled, chronically ill, or those suffering from systemic diseases.

5. After tooth loss, if patients are concerned about time, cost, and pain, and then fail to perform treatment, additional costs may occur due to changes in the position of various teeth in the oral cavity. In particular, there may be additional costs depending on whether or not a bone graft is received.

6. Dental implants are smaller than natural teeth. In the case of maxillary molars, there are three roots, but only one implant fixture. Therefore, the crown cannot be made as large as the molars. In addition, since the space is narrow when the interlocking teeth come down or come up, implant teeth are made smaller in size than natural teeth for various reasons.

7. Each doctor has different skills depending on his/her education and experience.

8. Although it is stable, there are cases in which an allergic reaction of unknown cause due to titanium etc. occurs severely throughout the body. Nor is it permanent. and others

Keywords: Disadvantages of implant, natural teeth, histological, anatomical

Acknowledgement: This study is supported by Uniance's Central Research Institute.

Copyright (C) 2021. Korean Academy of Preventive Dentistry. All rights reserved.

This is an Open Access article distributed under the terms of the Creative Commons Attribution Non-Commercial License (http://creativecommons.org/licenses/ by-nc/4.0) which permits unrestricted non-commercial use, distribution, and reproduction in any medium, provided the original work is properly cited. 